\begin{tabular}{|c|l|}
\hline Title & Realization of conductive InA s nanotubes based on lattice mismatched InP/nA s core shell nanowires \\
\hline Author(s) & Mohan, Premila; Motohisa, Junichi; Fukui, Takashi \\
\hline Citation & $\begin{array}{l}\text { A pplied Physics Letters, 88, 013110 } \\
\text { https://doi.org/_0.1063/2161576 }\end{array}$ \\
\hline Issue Date & 2006-01-02 \\
\hline Doc URL & http://hdl.handle.net/2115/8418 \\
\hline Rights & Copyright $\odot$ 2006 A merican Institute of Physics \\
\hline Type & article \\
\hline File Information & A pplPhysLett_88_013110.pdf \\
\hline
\end{tabular}

Instructions for use 


\title{
Realization of conductive InAs nanotubes based on lattice-mismatched InP/InAs core-shell nanowires
}

\author{
Premila Mohan, ${ }^{\text {a) }}$ Junichi Motohisa, and Takashi Fukui \\ Research Center for Integrated Quantum Electronics (RCIQE), Graduate School of Information Science \\ and Technology, Hokkaido University, North 13 West 8, Sapporo 060-8628, Japan
}

(Received 15 September 2005; accepted 6 December 2005; published online 5 January 2006)

\begin{abstract}
We report the realization of ordered arrays of single-crystalline InAs nanotubes by a simple pure-eptiaxial approach. The process involved the fabrication of lattice-mismatched InP/InAs core-shell nanowires using selective area metalorganic vapor phase epitaxy on InP (111)A substrates. The subsequent removal of the InP core resulted in vertically aligned InAs nanotubes which were highly uniform with well-defined features and controllable dimensions. Transmission electron microscopy studies confirmed that the nanotubes were single-crystalline with wurtzite crystal structure and temperature-dependent transport measurements revealed that they were conductive without any intentional doping. The realization of such conductive InAs nanotubes opens up new possibilities for both fundamental studies and future device applications. (C) 2006 American Institute of Physics. [DOI: 10.1063/1.2161576]
\end{abstract}

Nanoscale tubular structures have attracted extensive interest because of their exceptional physical properties and potential applications in nanoelectronics. ${ }^{1}$ Since the advent of carbon nanotubes, extensive research efforts have been carried out to synthesize nanoscale tubular structures based on materials other than carbon. Substances possessing layered crystal structure have been shown to form nanotubes under favorable conditions. ${ }^{2-4}$ Nanotubes from materials without layered crystal structure ${ }^{5-9}$ have also been synthesized using various methods, among which, singlecrystalline nanotubes based on semiconductor materials could serve as ideal building blocks for future miniaturized electronics. However, the feasibility of employing nanotubes as device building blocks in practical technological applications critically requires them to be electrically conductive. The major obstacle being posed is that most of the semiconductor materials become insulating at the nanometer scale due to depletion of carriers and further, doping onedimensional nanostructures still remains a concern due to the existence of random dopant fluctuations. ${ }^{10}$ A potential solution to circumvent this obstacle is to employ InAs, which is an exceptional material with superior electrical properties owing to its Fermi level pinning in the conduction band, thereby affording the exciting possibility to realize conductive semiconductor nanotubes. In addition, InAs has excellent properties such as narrow band gap, low electron effective mass, high-electron mobility, and high-saturation drift velocity which makes them extremely promising for nanoscale electronic, optoelectronic, and more particularly, low power and ultra high-speed device applications. In spite of its immense significance, InAs nanotubes have not been realized so far and the fabrication still remains challenging because of the lack of suitable lattice-matched substrate or heterostructure to facilitate the design and fabrication of the desired InAs nanotube.

In this letter, we report the fabrication of singlecrystalline conductive InAs nanotubes. Our applicationoriented approach was based on a single-step epitaxial

\footnotetext{
a) Author to whom correspondence should be addressed; electronic mail: pmohan@ rciqe.hokudai.ac.jp
}

growth of radial heterostructure core-shell nanowire and the subsequent removal of the inner core, which is similar to the recently reported AlGaAs nanotube fabrication. ${ }^{9}$ However, in case of AlGaAs nanotubes the core-shell nanowire consisted of the lattice-matched $\mathrm{GaAs}-\mathrm{AlGaAs}$ material system, whereas herein, the core-shell nanowire should comprise of lattice-mismatched materials as there is essentially no latticematched material that could serve as the core to the InAs shell. In the present work, we chose the InP/InAs material system which has a lattice-mismatch of $3.22 \%$. Due to this lattice-mismatch, the growth of InAs over InP usually results in the formation of InAs three-dimensional islands via Stranski-Krastanow (SK) growth mode. While there are reports on axial heterostructure nanowires comprising of InP segments embedded in InAs nanowires by the vapor-liquidsolid (VLS) technique, ${ }^{11}$ there is virtually no report on InP/InAs core-shell nanowires. In the present work, we employed the catalyst-free selective area metalorganic vapor phase epitaxy (SA-MOVPE) which is a totally different approach to the conventional metal-catalyst assisted VLS growth technique by which most of the nanowires are grown. ${ }^{12}$ The advantages of SA-MOVPE are that the nanowires can be grown without the use of a seed particle and their size, shape, and position can be precisely controlled. $^{13-15}$ Also, nanotubes realized from core-shell nanowires grown by SA-MOVPE will be of superior crystal-

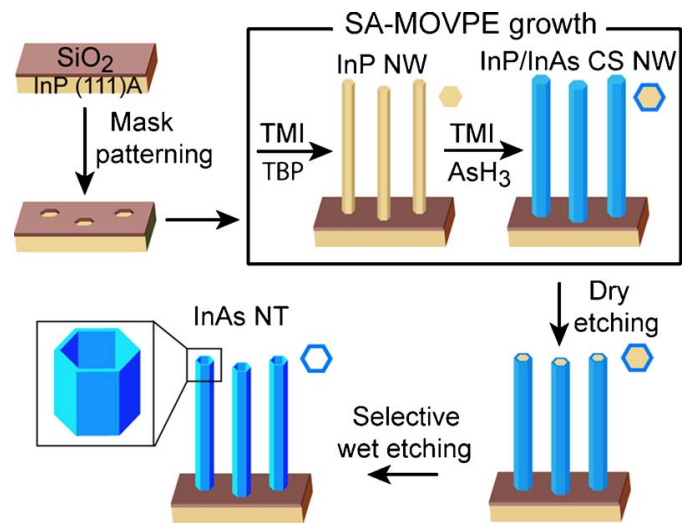

FIG. 1. Fabrication sequence of InAs nanotube periodic arrays. 


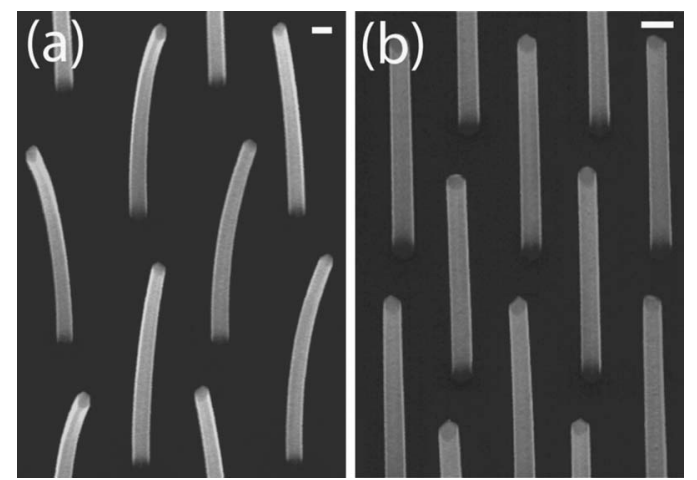

FIG. 2. SEM images of (a) bent and (b) vertically erect InP/InAs core-shell nanowires fabricated by SA-MOVPE (scale bars $100 \mathrm{~nm}$ ).

line quality as both the core and the shell are grown by pure epitaxial method and the catalyst-free, single-step growth will reduce defects and other unwanted impurity incorporation.

The fabrication sequence of InAs nanotube array is shown in Fig. 1. The mask pattern consisted of hexagonal

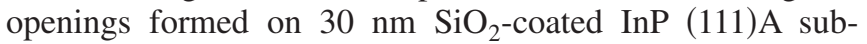
strates by electron beam lithography and wet chemical etching. SA-MOVPE growth of InP/InAs core-shell nanowires was carried out as a single-step process using a low-pressure horizontal MOVPE system and the source materials were trimethylindium (TMI), tertiarybutylphosphine (TBP), and $5 \%$ arsine $\left(\mathrm{AsH}_{3}\right)$ in hydrogen. At first, InP nanowire growth was initiated and after substantial growth, the source materials and growth conditions were altered accordingly to grow the InAs layer. For InP growth, the growth temperature was $625^{\circ} \mathrm{C}$, partial pressures of TMI and TBP were $4.4 \times 10^{-6}$ and $1.65 \times 10^{-4} \mathrm{~atm}$, respectively and growth time was 20 $\min$. The InP nanowires grown under these conditions were of diameter $70 \mathrm{~nm}$ and length $2 \mu \mathrm{m}$, and possess hexagonal cross section with distinct $\{110\}$ vertical facets, the growth mechanism of which is discussed in detail elsewhere. ${ }^{15} \mathrm{Sev}-$ eral experiments were carried out to determine the optimum conditions that could favor layer-by-layer epitaxial growth of InAs over InP core despite the lattice mismatch, and that too preferentially along the lateral $\langle 110\rangle$ direction. Systematic studies revealed that the growth mode of InAs over InP depends on a delicate balance of various factors governed by strain related thermodynamics and growth kinetic effects. Growth temperature was found to have a strong influence on the growth mode and at $510{ }^{\circ} \mathrm{C}$, InAs layer-by-layer growth via Frank-van der Merwe growth mode occurred along the InP nanowire sidewall facets thus resulting in the successful fabrication of $\mathrm{InP} / \mathrm{InAs}$ core-shell nanowires. However, the resultant nanowires were observed to be bent in a random manner [Fig. 2(a)]. Extensive analysis of the bent nanowires indicated that the lateral growth of the InAs layer over InP core was not uniform and that the InP core was partially exposed along the sidewalls. This non uniform growth of the outer InAs layer combined with the built-in strain of the lattice-mismatched InP/InAs epitaxial layers may be attributed to the bending of the core-shell nanowires. Further experiments revealed that low InAs growth rate was the essential factor to realize uniform InAs shell growth over InP core. Growth temperature of $400{ }^{\circ} \mathrm{C}$ and TMI and $\mathrm{AsH}_{3}$ partial pressures of $4.89 \times 10^{-7}$ and $6.25 \times 10^{-5} \mathrm{~atm}$, respectively, were found to be the optimum conditions that resulted in
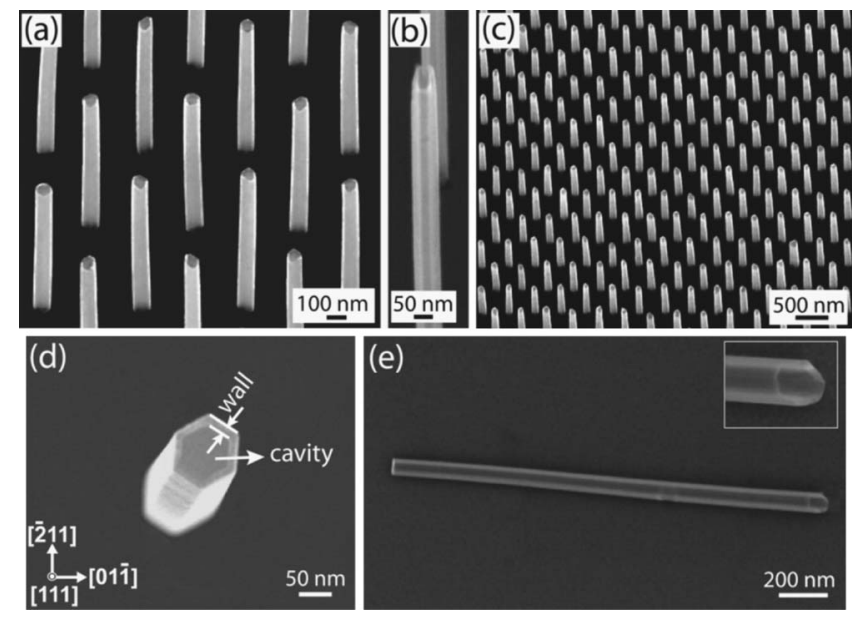

FIG. 3. SEM images of InAs nanotube periodically aligned arrays. (a) Vertically aligned InAs nanotube ordered array on InP (111)A substrate. (b) Magnified image of a single vertical standing InAs nanotube showing its tubular nature. (c) Low-angle view of a typical high-dense nanotube array. (d) Low-angle inclined, high-resolution image revealing the hexagonal cross section and sixfold symmetric $\{110\}$ vertical facets (e) Horizontal lying InAs nanotube with top end closed in which case the InP core was selectively etched from the bottom end. The inlet shows the magnified image of the tube opening.

uniform and vertically erect $\mathrm{InP} / \mathrm{InAs}$ core-shell nanowires [Fig. 2(b)]. The nanowires were periodically aligned and exhibited hexagonal cross section with sixfold symmetric $\{110\}$ vertical sidewall facets. The core-shell nanowires were then subjected to a two-step etching process to realize the InAs nanotubes. Since the grown nanowires were capped by a thin InAs layer, anisotropic dry etching involving pure Ar-ion sputtering was employed to remove their tip in order to expose the inner InP core at the top end. Subsequently, selective wet etching using $\mathrm{HCl} / \mathrm{H}_{3} \mathrm{PO}_{4}$ solution was carried out to remove the inner core which resulted in vertical-standing InAs nanotubes.

Scanning electron microscopy (SEM) studies revealed that the nanotubes were highly uniform with well-defined size and shape [Figs. 3(a)-3(e)]. The length of the nanotubes was $2 \mu \mathrm{m}$ with an inner diameter of $70 \mathrm{~nm}$ and wall thickness of $10 \mathrm{~nm}$. The remarkable feature is that these dimensions were precisely controllable and tunable by careful choice of growth conditions thereby enabling the possibility to realize nanotubes with tailored properties. While the length depends on the growth time of the InP core, the inner diameter can be controlled by the mask opening width and InP growth parameters. Nanotube thickness was found to directly depend on the InAs growth time, making use of which nanotubes with different thickness were fabricated. Both the inner and outer walls of the nanotubes exhibited perfect hexagonal cross section with distinct sixfold symmetric $\{110\}$ vertical facets normal to the (111)A plane. The lithography defined mask patterning and high selectivity provided site control of individual nanotubes resulting in the formation of highly uniform, periodic arrays of InAs nanotubes. These periodically aligned and vertically oriented nanotubes can eliminate the difficulty of the lithographic steps involved in pick-and-place approach and thus can facilitate the bottom-up assembly of three-dimensional complex nanostructures by utilizing the recently emerged process technologies to integrate as-grown one-dimensional nanostructures into devices. ${ }^{16}$ 

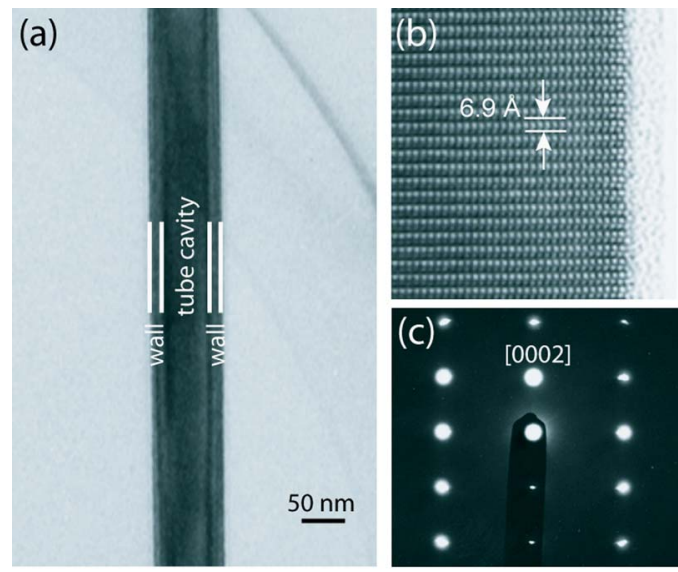

(d)

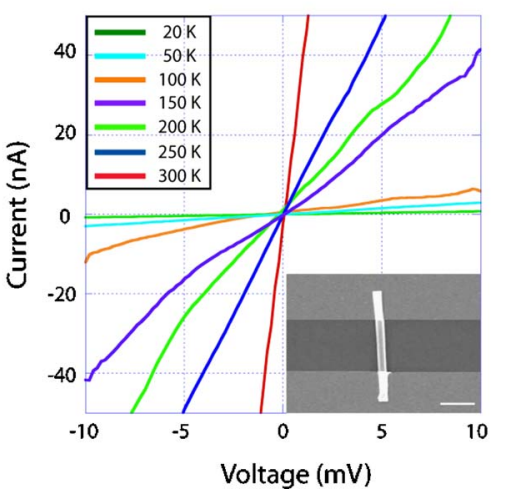

FIG. 4. (a) TEM, (b) high-resolution TEM (HRTEM) images, and (c) electron diffraction pattern of a typical InAs nanotube. (d) Temperaturedependent $I-V$ curves of an undoped InAs nanotube. SEM image of electrically contacted nanotube is shown in the inset (scale bar $1 \mu \mathrm{m}$ ).

The grown nanotubes were subjected to transmission electron microscopy (TEM) for further structural analysis. TEM analysis indicated that the InAs nanotubes were of tubular nature with uniform wall thickness [Fig. 4(a)]. The diameter and wall thickness of the nanotubes observed using TEM were consistent with the SEM studies. The lattice resolved data and electron diffraction measurements confirmed the single crystallinity of the grown nanotubes and the crystal structure was hexagonal wurtzite rather than the usual zinc-blende structure [Figs. 4(b) and 4(c)]. In order to find the origin of this wurtzite crystal structure, TEM analysis was carried out on InP as well as InP/InAs core-shell nanowires and both exhibited wurtzite structure. In general, nanowires grown along the $\langle 111\rangle$ direction are either zinc blende with a high concentration of stacking faults, or polytypes with intermixed zinc blende and wurtzite structures. ${ }^{17}$ But in the present case, the $\langle 111\rangle$ A-grown InP nanowires were found to be purely wurtzite. This may be due to the perfect periodic occurrence of a rotational twin or intrinsic stacking fault on every third $\{111\}$ layer of the zinc-blende InP. As the epitaxial growth of InAs proceeds over InP, the crystal structure continues across the heterointerface thereby resulting in wurtzite crystal structure for InP/InAs core-shell nanowire and the resultant InAs nanotube. The lattice constant $c$ of the nanotube was found to be $6.9 \AA$ which is in agreement with the theoretical calculations for bulk InAs. ${ }^{18}$ To characterize the electrical properties of the grown nanotubes, temperature-dependent two-terminal transport measurements on individual nanotubes were performed. Figure 4(d) shows the typical temperature-dependent current-voltage $(I-V)$ curves obtained from individual undoped InAs nanotube. The nanotubes exhibited good ohmic characteristics from room temperature down to $20 \mathrm{~K}$. The two-terminal resistance at room temperature was as low as $25 \mathrm{k} \Omega$ and the resistance was found to increase with a decrease in temperature. Thus the results evidently indicate that the nanotubes were electrically conductive without any intentional doping. Such conductivity in InAs nanotubes could be attributed to the effect of the Fermi level pinning in the conduction band because of which there is an accumulation of carriers at the surface. While in other semiconductors, the surface Fermi level is pinned in the band gap resulting in surface depletion, the presence of the surface accumulation layer in InAs makes it electrically conductive even for nanometer scale structures without any intentional doping. ${ }^{19}$

In summary, we have fabricated single-crystalline, conductive InAs nanotubes based on lattice-mismatched InP/InAs core-shell nanowires grown by selective area MOVPE. The realized nanotubes were highly uniform, vertically oriented, and reproducibly identical with well-defined features and controllable dimensions and with perfect positioning in predetermined configurations. The nanotubes were single crystalline with wurtzite crystal structure and the undoped nanotubes exhibited electrical conductivity. Realization of such conductive nanotubes based on a conventional semiconductor material with controllable and tunable features offers significant potential for a wide range of innovative nanodevice applications.

The authors thank T. Hashizume, S. Hara, J. Takeda, N. Ooike, J. Noborisaka, and T. Oikawa for discussions and S. Akamatsu for experimental support. One of the author (P.M.) was supported by the Japan Society for Promotion of Science postdoctoral fellowship.

${ }^{1}$ A. Bachtold, P. Hadley, T. Nakanishi, and C. Dekker, Science 294, 1317 (2001).

${ }^{2}$ R. Tenne, L. Margulis, M. Genut, and G. Hodes, Nature (London) 360, 444 (1992).

${ }^{3}$ N. G. Chopra, R. J. Luyken, K. Cherrey, V. H. Crespi, M. L. Cohen, S. G. Louie, and A. Zettl, Science 269, 966 (1995).

${ }^{4}$ P. M. Ajayan, O. Stephan, Ph. Redlich, and C. Colliex, Nature (London) 375, 564 (1995).

${ }^{5}$ R. Martin, Science 266, 1961 (1994).

${ }^{6}$ B. Lakshmi, C. J. Patrissi, and C. R. Martin, Chem. Mater. 9, 2544 (1997).

${ }^{7}$ J. Goldberger, R. He, Y. Zhang, S. Lee, H. Yan, H. J. Choi, and P. Yang, Nature (London) 422, 599 (2003).

${ }^{8}$ E. P. A. M. Bakkers, and M. A. Verheijen, J. Am. Chem. Soc. 125, 3440 (2003).

${ }^{9}$ J. Noborisaka, J. Motohisa, S. Hara, and T. Fukui, Appl. Phys. Lett. 87, 093109 (2005).

${ }^{10}$ L. J. Lauhon, M. S. Gudiksen, and C. M. Lieber, Philos. Trans. R. Soc. London, Ser. A 362, 1247 (2004).

${ }^{11}$ M. T. Bjork, B. J. Ohlsson, T. Sass, A. I. Persson, C. Thelander, M. H. Magnusson, K. Deppert, L. R. Wallenberg, and L. Samuelson, Appl. Phys. Lett. 80, 1058 (2002).

${ }^{12}$ K. Hiruma, M. Yazawa, K. Haraguchi, K. Ogawa, T. Katsuyama, M. Koguchi, and H. Kakibayashi, J. Appl. Phys. 74, 3162 (1993).

${ }^{13}$ J. Motohisa, J. Noborisaka, J. Takeda, M. Inari, and T. Fukui, J. Cryst. Growth 272, 180 (2004).

${ }^{14}$ J. Noborisaka, J. Motohisa, and T. Fukui, Appl. Phys. Lett. 86, 213102 (2005).

${ }^{15}$ P. Mohan, J. Motohisa, and T. Fukui, Nanotechnology 16, 2903 (2005).

${ }^{16}$ T. N. Hou, J. Han, T. Yamada, P. Nguyen, Y. P. Chen, and M. Meyyappan, Nano Lett. 4, 1247 (2004).

${ }^{17}$ K. Hiruma, M. Yazawa, T. Katsuyama, K. Ogawa, K. Haraguchi, M. Koguchi, and H. Kakibayashi, J. Appl. Phys. 77, 447 (1995).

${ }^{18}$ S. Q. Wang, and H. Q. Ye, J. Phys.: Condens. Matter 14, 9579 (2002).

${ }^{19}$ H. Yamaguchi, J. L. Sudijono, B. A. Joyce, T. S. Jones, C. Gatzke, and R. A. Stradling, Phys. Rev. B 58, 4219 (1998). 